\title{
Avaliação dos parâmetros de secagem da polpa de graviola em pó obtida por secagem em spray dryer
}

\author{
Evaluation of the drying parameters of soursop pulp powder obtained by \\ drying in a spray dryer
}

\author{
Carlos Eliardo Barros Cavalcante ${ }^{1 *}$, Sueli Rodrigues ${ }^{2}$, Marcos Rodrigues Amorim Afonso², José Maria Correia Costa ${ }^{2}$ \\ 'Instituto Federal de Educação, Ciência e Tecnologia do Ceará (IFCE), Ubajara/CE - Brasil \\ ${ }^{2}$ Universidade Federal do Ceará (UFC), Centro de Ciências Agrárias, Departamento de Tecnologia de Alimentos, Campus do Pici, Fortaleza/CE - Brasil
}

\section{*Corresponding Author}

Carlos Eliardo Barros Cavalcante, Instituto Federal de Educação, Ciência e Tecnologia do Ceará (IFCE), Campus Ubajara, Rua Luiz Cunha, s/n, Monte Castelo, CEP: 62350-000, Ubajara/CE - Brasil, e-mail: eliardo@ifce.edu.br

Cite as: Evaluation of the drying parameters of soursop pulp powder obtained by drying in a spray dryer. Braz. J. Food Technol., v. 20, e2016015, 2017.

Received: Jan. 22, 2016; Accepted: May 22, 2017

\section{Resumo}

A polpa de graviola é um produto amplamente consumido no Brasil e sua desidratação pelo processo spray dryer é alternativa relevante para aproveitamento do excedente de produção. Dentro deste contexto, este trabalho teve como objetivo estabelecer a melhor condição operacional de secagem em spray dryer, visando a obtenção de polpa de graviola em pó por meio do planejamento composto central rotacional, tendo a concentração de maltodextrina e a temperatura do ar de secagem como variáveis independentes. Verificaram-se os efeitos negativos lineares e quadráticos significativos $(p<0,05)$ da concentração de maltodextrina e da temperatura do ar de secagem sobre a umidade da polpa de graviola em pó. Apesar de o modelo não se ajustar aos parâmetros de higroscopicidade e rendimento, foi possível, através do teste de médias, estabelecer as condições de processo. Considerando-se o uso de baixas temperaturas e menores concentrações de maltodextrina, recomenda-se o emprego de $169^{\circ} \mathrm{C}$ como temperatura de secagem e $17 \%(\mathrm{~m} / \mathrm{m})$ de maltodextrina como adjuvante de secagem. $\mathrm{O}$ uso de tais condições acarreta um produto com baixos teores de umidade e atividade de água, classificado como ligeiramente higroscópico, suscetível ao caking, e que apresenta microestruturas com formato esférico de tamanhos variados e de superfície lisa e uniforme.

Palavras-chave: Polpa de fruta; Atomização; Metodologia de superfície de resposta; Higroscopicidade; Microestruturas.

\section{Abstract}

Soursop pulp is a widely consumed product in Brazil and its dehydration by spray drying has been shown to be a relevant alternative for the exploitation of surplus production. Thus the objective of this research was to establish the best operational conditions to dry soursop pulp in a spray dryer to obtain the powder, using a central composite rotational design (CCRD), the independent variables being the maltodextrin concentration and the drying air temperature. Significant negative linear and quadratic effects $(p<0.05)$ were found for the maltodextrin concentration and drying air temperature on the moisture content of the powdered soursop pulp. Although the model did not adjust to the hygroscopicity and yield parameters, the process conditions could be established using the means test. Considering the use of low temperatures and lower maltodextrin concentrations, the use of a drying temperature of $169{ }^{\circ} \mathrm{C}$ and $17 \%(\mathrm{w} / \mathrm{w})$ of maltodextrin as the drying aid was recommended. The use of such conditions resulted in a product with low moisture content and water activity, classified as slightly hygroscopic, caking sensitive and that presents a microstructure with spherical shape, different sizes and smooth and uniform surface.

Keywords: Fruit pulp; Spray drying; Response surface methodology; Hygroscopicity; Microstructures. 


\section{Introdução}

A graviola é uma fruta tropical que apresenta alto valor comercial no Brasil, com grandes perspectivas econômicas para comercialização e exportação. (SÃO JOSÉ et al., 2014). Por apresentar excelentes características sensoriais e valor nutritivo significativo, os produtores apostam cada vez mais na sua industrialização para produção de polpas, sucos e néctares (LEMOS, 2014; WATANABE et al., 2014).

A secagem por aspersão em spray dryer é um processo amplamente utilizado na indústria de alimentos e, em condições ideais, tem se mostrado eficaz para a obtenção de diversos produtos (ROCHA et al., 2014). É um processo contínuo, que se caracteriza pela transformação de um líquido em um produto seco, na forma de pó, por meio de um tempo de secagem muito curto. Nesse processo, o líquido é aspergido, utilizando-se um sistema de alta pressão, pelo qual as gotículas entram em contato com um fluxo de ar quente. Assim, há uma rápida evaporação, que permite manter baixa a temperatura do produto final, possibilitando a secagem de produtos sensíveis ao calor sem afetar excessivamente sua qualidade (FERRARI et al., 2012).

Entretanto, é um processo de difícil utilização em alimentos ricos em açúcares, devido à obtenção de produtos com alta higroscopicidade, que minimizam o rendimento do processo, bem como o escoamento e a reconstituição desses alimentos em pó. Portanto, é fundamental a utilização de temperaturas mais baixas no processo de secagem, bem como o emprego de agentes carreadores com alto peso molecular antes da secagem, visando facilitar e aumentar o rendimento do processo (OLIVEIRA et al., 2007; FERRARI et al., 2012). A maltodextrina é o agente carreador comumente empregado na secagem por aspersão em spray dryer, em função de sua alta solubilidade, baixa higroscopicidade e baixo custo (KHA et al., 2010).

Neste contexto, o objetivo deste trabalho foi estudar a influência da temperatura do ar de secagem e da concentração de maltodextrina sobre as características da polpa de graviola em pó, produzida por secagem por aspersão em spray dryer, visando à determinação das condições de processamento, além de caracterizar físico-química e morfologicamente a polpa em pó obtida.

\section{Material e métodos}

As polpas de graviola - não pasteurizadas e sem adição de conservantes - selecionadas para a realização deste estudo foram adquiridas em uma indústria de processamento de polpas de frutas localizada no município de Fortaleza-CE e encaminhadas para o Laboratório de Controle de Qualidade de Alimentos e Secagem do Departamento de Tecnologia de Alimentos da Universidade Federal do Ceará, onde foram armazenadas à temperatura de $-18{ }^{\circ} \mathrm{C}$ e utilizadas de acordo com a necessidade experimental.

O descongelamento foi realizado com as polpas ainda embaladas sob temperatura de $6 \pm 2{ }^{\circ} \mathrm{C}$, em refrigerador, por um período mínimo de 18 horas. Utilizou-se maltodextrina com dextrose equivalente (DE) 20 como coadjuvante de secagem na formulação da polpa de graviola para secagem.

A secagem da polpa foi realizada em equipamento spray dryer modelo LM SMD 1.0 da marca Labmaq do Brasil, Ribeirão Preto-SP, com a utilização de um bico aspersor de 1,2 mm de diâmetro. Durante os processos de aspersão, a temperatura de secagem e a concentração de maltodextrina sofreram variações, de acordo com o planejamento experimental. Foram mantidos constantes os parâmetros de vazão do ar comprimido (3,0 L/min), pressão de aspersão (100 psi), vazão de alimentação (0,5 L/h) e vazão do ar de secagem (3,5 m³/min).

Para avaliar a influência da temperatura de secagem e da concentração de maltodextrina na polpa sobre o pó obtido no processo, foi realizado um delineamento do tipo composto central rotacional $2^{2} \mathrm{com}$ três repetições no ponto central (Tabela 1), tendo como variáveis respostas a umidade e a higroscopicidade do pó. As faixas de variação entre os limites inferior e superior para variáveis independentes foram estabelecidas a partir de dados da literatura e de testes preliminares realizados.

Para cada ensaio experimental, foram desidratados, aproximadamente, $400 \mathrm{~g}$ de polpa de graviola formulada com maltodextrina em percentual $(\mathrm{m} / \mathrm{m})$ determinado, com base nos resultados do planejamento experimental. Os processos de secagem foram conduzidos de maneira o mais uniforme possível, para que não houvesse diferença entre as condições de trabalho.

A relação entre as variáveis independentes e as variáveis respostas foi estabelecida através de modelos matemáticos. O modelo utilizado foi a equação de segunda ordem (Equação 1):

$$
\mathrm{Y}=\beta_{0}+\beta_{1} \mathrm{X}_{1}+\beta_{2} \mathrm{X}_{2}+\beta_{11} \mathrm{X}_{1}^{2}+\beta_{22} \mathrm{X}_{2}^{2}+\beta_{12} \mathrm{X}_{1} \mathrm{X}_{2}+\text { Error }
$$

em que: $Y$ = variável reposta; $\beta_{0}=$ intersecção (constante); $X_{1}$ e $X_{2}=$ variáveis independentes (temperatura e concentração

Tabela 1. Níveis do planejamento experimental para secagem da polpa de graviola em spray dryer.

\begin{tabular}{lccccc}
\multicolumn{1}{c}{ Variáveis independentes } & \multicolumn{4}{c}{ Níveis } \\
\cline { 2 - 6 } & $\mathbf{- 1 , 4 1}$ & $\mathbf{- 1 , 0}$ & $\mathbf{0}$ & $\mathbf{+ 1 , 0}$ & $\mathbf{+ 1 , 4 1}$ \\
Temperatura de secagem $\left({ }^{\circ} \mathrm{C}\right)$ & 148 & 154 & 169 & 184 & 190 \\
Concentração de maltodextrina $(\% \mathrm{~m} / \mathrm{m})$ & 10 & 12 & 17 & 22 & 24 \\
\hline
\end{tabular}


de maltodextrina); $\beta_{1}, \beta_{2}, \beta_{11}, \beta_{22}, \beta_{12}=$ coeficientes da regressão (linear, quadrático e interação).

Após a análise estatística dos coeficientes, a análise de variância (ANOVA) foi aplicada com o intuito de testar a adequação dos modelos gerados através da avaliação do coeficiente de determinação $\left(R^{2}\right)$ e do teste F. Os termos que não eram estatisticamente significativos $(p>0,05)$ foram excluídos do modelo básico e reajustados. Os coeficientes de determinação $\left(R^{2}\right)$, a determinação do coeficiente ajustado $\left(\mathrm{R}^{2}{ }_{\text {adj }}\right)$ e a análise da falta de ajuste foram usados para avaliar a qualidade dos novos modelos, sendo as parcelas de superfície geradas.

Para os modelos que apresentaram falta de ajuste significativo, foi realizado o teste de Tukey, para comparação entre as médias $(\alpha=0,05)$, visando maior fundamentação na determinação das condições que geram polpa de graviola em pó com melhores parâmetros de umidade e higroscopicidade. Os dados foram tratados estatisticamente com o auxílio do software Statistica 7.0 (STATSOFT, 2007), de forma a assegurar a validade dos coeficientes dentro de um intervalo de confiança de 95\%.

Todas as determinações físico-químicas, tanto na polpa integral quanto na polpa em pó obtida na melhor condição de processo, foram realizadas em triplicata. As análises foram as seguintes: atividade de água, determinada a $25{ }^{\circ} \mathrm{C}$ em higrômetro digital (Aqualab ${ }^{\circledR}$ 3TE, Decagon, Pullman), e umidade, determinada por infravermelho em determinador de umidade digital (ID50, Marte, São Paulo).

$\mathrm{O} \mathrm{pH}$ foi determinado através de um medidor de $\mathrm{pH}$ (Q400A, Quimis, São Paulo), calibrado periodicamente com soluções tampão de $\mathrm{pH}$ 4,0 e 7,0, e a acidez titulável, por titulação com $\mathrm{NaOH} 0,1 \mathrm{~N}$, com os resultados expressos em percentagem de ácido cítrico e ácido málico. Os sólidos solúveis foram determinados através da leitura dos ${ }^{\circ}$ Brix a $20^{\circ} \mathrm{C}$, em refratômetro com escala variando de 0 a $90^{\circ} \mathrm{Brix}$ ( $r^{2}$ mini, Reichert, Nova York), segundo a metodologia do IAL (2005).
O conteúdo de vitamina $\mathrm{C}$ foi determinado por método titulométrico, baseado na redução do indicador 2,6-diclorofenolindofenol pelo ácido ascórbico. Os resultados foram expressos como: mg de ácido ascórbico/100 g de amostra (CUNNIFF, 1997).

A análise de cor foi determinada utilizando-se colorímetro (Chroma-Meter CR-410, Konica Minolta, Japão) com determinação da escala $\mathrm{CIE} \mathrm{L}^{*} \mathrm{a}^{*} \mathrm{~b}^{*}$, que inclui as variáveis $\mathrm{L}^{*} \mathrm{a}^{*} \mathrm{~b}^{*}$, Chroma $\left(\mathrm{C}^{*}\right)$ e ângulo Hue $\left(\mathrm{H}_{0}^{*}\right)$.

A determinação da densidade aparente foi realizada segundo metodologia proposta por Goula e Adamopoulos (2010), com resultados expressos em g/mL.

A caracterização higroscópica da polpa de graviola em pó obtida na melhor condição de processo foi realizada por meio das determinações de higroscopicidade (GOULA; ADAMOPOULOS, 2010), expressa em percentual de umidade adsorvida; grau de caking (JAYA; DAS, 2004) e solubilidade (CANO-CHAUCA et al., 2005), ambos expressos em percentual, e tempo de reidratação (GOULA; ADAMAPOULOS, 2010), expresso em segundos, para a amostra ser completamente reidratada.

A análise morfológica e da superfície dos pós foi realizada em microscópio eletrônico de varredura (MEV) (DSM940A, Zeiss, Oberkochen). Os pós foram depositados sobre fita adesiva dupla face, fixada em suporte metálico. A placa metálica foi recoberta com platina em metalizadora da marca Emitech, modelo K 550, operando a 5 m e $10 \mathrm{kV}$, fornecendo uma cobertura de aproximadamente $25 \mu \mathrm{m}$, ocorrendo, então, a captura das imagens.

\section{Resultados e discussão}

No planejamento composto central rotacional, utilizando-se, como variáveis independentes, a temperatura de secagem e a concentração de maltodextrina $(\% \mathrm{~m} / \mathrm{m})$, foram observados resultados médios para as variáveis de higroscopicidade e umidade, que são apresentados na Tabela 2.

Tabela 2. Planejamento composto central rotacional utilizado para a secagem em spray dryer e resultados médios observados para os parâmetros de higroscopicidade e umidade.

\begin{tabular}{ccccc} 
Ensaio & Temperatura $\left({ }^{\circ} \mathbf{C}\right)$ & Maltodextrina $(\% \mathbf{~ m} / \mathbf{m})$ & Higroscopicidade $(\% \mathbf{~ m} / \mathbf{m})$ & Umidade $(\% \mathbf{~ m} / \mathbf{m})$ \\
1 & $184(+1)$ & $22(+1)$ & $10,62 \pm 1,19^{\mathrm{abc}}$ & $0,90 \pm 0,04$ \\
2 & $154(-1)$ & $22(+1)$ & $9,79 \pm 1,24^{\mathrm{bc}}$ & $1,16 \pm 0,09$ \\
3 & $184(+1)$ & $12(-1)$ & $9,88 \pm 0,30^{\mathrm{bc}}$ & $1,34 \pm 0,17$ \\
4 & $154(-1)$ & $12(-1)$ & $8,14 \pm 1,28^{\mathrm{c}}$ & $1,43 \pm 0,29$ \\
5 & $148(-1,41)$ & $17(0)$ & $7,56 \pm 0,48^{\mathrm{c}}$ & $1,45 \pm 0,05$ \\
6 & $190(+1,41)$ & $17(0)$ & $13,12 \pm 2,67^{\mathrm{a}}$ & $0,95 \pm 0,01$ \\
7 & $169(0)$ & $10(-1,41)$ & $11,41 \pm 0,16^{\mathrm{ab}}$ & $1,45 \pm 0,29$ \\
8 & $169(0)$ & $24(+1,41)$ & $10,07 \pm 0,67^{\mathrm{abc}}$ & $0,88 \pm 0,16$ \\
9 & $169(0)$ & $17(0)$ & $10,30 \pm 0,45^{\mathrm{abc}}$ & $1,11 \pm 0,08$ \\
10 & $169(0)$ & $17(0)$ & $10,50 \pm 0,16^{\mathrm{abc}}$ & $1,09 \pm 0,07$ \\
\hline
\end{tabular}

Letras diferentes na mesma coluna indicam diferença significativa entre os tratamentos para $p \leq 0,05$. 
A temperatura de secagem e a concentração de maltodextrina, nos níveis utilizados apresentaram falta de ajuste $(p<0,05)$ sobre a higroscopicidade da polpa de graviola em pó obtida por secagem em spray dryer. Portanto, não foi possível obter modelos matemáticos.

A higroscopicidade dos pós de polpa de graviola variou de $7,56 \%$ a $13,12 \%$. De acordo com a classificação proposta por GEA Niro Research Laboratory (2003), os pós de polpa de graviola obtidos nas condições utilizadas no planejamento são ligeiramente higroscópicos.

A provável explicação para variações na higroscopicidade dos pós pode estar relacionada com a faixa de temperatura utilizada no planejamento. De acordo com Tonon et al. (2008), temperaturas de secagem mais altas resultam em pós com umidades mais baixas e maior facilidade em adsorver água, ou seja, mais higroscópicos, o que está relacionado ao maior gradiente de concentração de água existente entre o produto e o ambiente. Na variação da higroscopicidade, pode-se observar que os ensaios correspondentes aos pontos centrais do planejamento - temperatura de $169{ }^{\circ} \mathrm{C}$ e $17 \%(\mathrm{~m} / \mathrm{m})$ de maltodextrina - não apresentaram diferença significativa em relação aos outros ensaios.

Em relação à umidade, o modelo obtido foi satisfatório $(p<0,05)$, mostrando falta de ajuste. A avaliação dos efeitos das variáveis independentes sobre os teores de umidades dos pós mostrou que apenas a interação temperatura de secagem versus concentração de maltodextrina não foi estatisticamente significativa. O efeito estimado para os fatores temperatura de secagem e concentração de maltodextrina em termos lineares foi negativo, ou seja, aumentos em quaisquer desses fatores resultam em pós mais secos.

A.

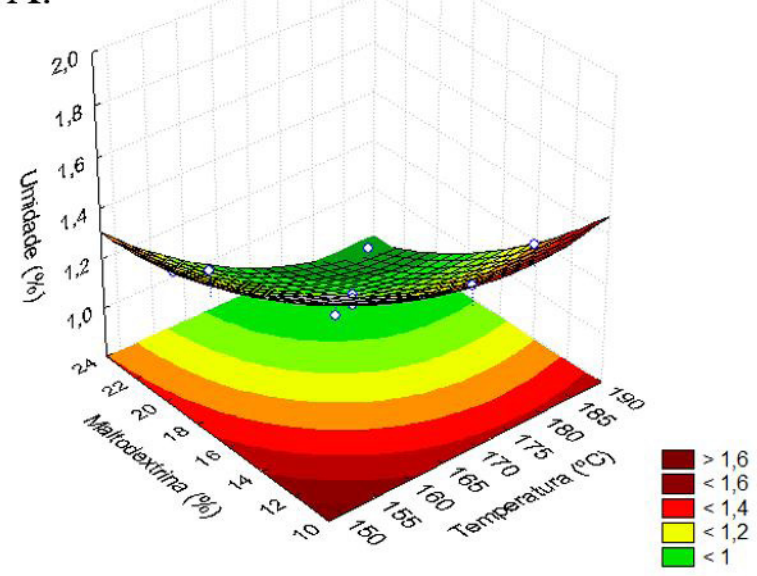

Na secagem em spray dryer, é comum observar que o aumento da temperatura de secagem resulta em maior perda de água do material a ser desidratado devido a uma maior taxa de transferência de calor sobre as partículas, promovendo grande força motriz para a evaporação de água, resultando, consequentemente, em pós com menor teor de umidade (FERRARI et al., 2012). Esse comportamento foi observado em diversos trabalhos, como os relatados por Quek et al. (2007), em estudo com suco de melancia; Moreira et al. (2009), com suco de acerola em pó; Tonon et al. (2009), com suco de açaí, e por Rocha et al. (2014), com suco de caju.

De acordo como Abadio et al. (2004) e Quek et al. (2007), a utilização de maltodextrina como coadjuvante de secagem promove um aumento no teor de sólidos totais e reduz o teor de umidade do material a ser desidratado, facilitando a obtenção de pós com menor teor de umidade. Tal efeito, como esperado e desejado, foi observado no presente estudo.

Uma vez que somente os termos lineares e quadráticos foram significativos, um novo modelo foi gerado com base nos coeficientes lineares de modo a não afetar a qualidade do modelo. O percentual de variação explicada pelo modelo foi maior que $96 \%$ e o valor de $\mathrm{F}$ calculado para o modelo foi de 33,59, ou seja, 7,41 vezes maior que o valor de $F$ tabelado $(4,53)$, no intervalo de $95 \%$ de confiança. Assim, o modelo gerado neste estudo pode ser considerado estatisticamente preditivo. Usando-se a Equação 2, foi possivel elaborar a superfície de resposta que mostra o comportamento das variáveis independentes sobre o teor de umidade da polpa de graviola em pó (Figura 1).

Umidade $(\%)=11,95-0,106 \mathrm{~T}+0,0003 \mathrm{~T}^{2}-0,102 \mathrm{M}+0,002 \mathrm{M}^{2}(2)$ em que: $R^{2}=0,97 ; R_{\text {ajust }}^{2}=0,96$ e $P$-valor [falta de juste] $=0,07$.

Figura 1. Superfície de resposta (A) e curva de contorno (B) para a variável resposta umidade em função das variáveis independentes (temperatura de entrada do ar e concentração de maltodextrina), para a polpa de graviola desidratada em spray dryer. 
Nenhum dos ensaios foi realizado na região ótima para a resposta avaliada, já que, de acordo com a Figura $1 \mathrm{~A}$ e 1B, seriam necessárias temperaturas mais altas e maiores concentrações de maltodextrina. No entanto, deve-se ressaltar que, devido a limitações do próprio equipamento, não seria possível trabalhar com temperaturas acima de $190^{\circ} \mathrm{C}$ e que, apesar de a superfície de resposta indicar tal tendência, temperaturas mais altas não seriam necessárias, pois foram obtidos resultados de umidade relativamente baixos e considerados aceitáveis para a estabilidade do pó.

Teores de umidade semelhantes aos obtidos neste estudo foram relatados por Ferrari et al. (2012), em estudo com suco de amora-preta, obtendo, com $15 \%$ de maltodextrina e temperaturas de $180{ }^{\circ} \mathrm{C}$ e $160{ }^{\circ} \mathrm{C}$, pós com $1,02 \%$ e $1,51 \%$ de umidade, respectivamente. Similarmente, Rocha et al. $(2013,2014)$, em estudo com polpa de manga e suco de caju desidratados com 30\% de maltodextrina a $178{ }^{\circ} \mathrm{C}$, obtiveram pós com teores de umidade de $1,04 \%$ e $1,58 \%$, respectivamente.

Diante do exposto, por intermédio do uso de temperaturas mais baixas e menores concentrações de maltodextrina, recomenda-se a secagem da polpa de graviola em spray dryer a uma temperatura de $169^{\circ} \mathrm{C}$ e $17 \%(\mathrm{~m} / \mathrm{m})$ de maltodextrina como agente carreador, uma vez que essas condições apresentaram maior rendimento, pós com umidade aceitável e higroscopicidade semelhante aos outros ensaios.

Na Tabela 3, encontram-se os resultados das análises físico-químicas realizadas na polpa integral de graviola e na polpa em pó obtida por secagem em spray dryer, à temperatura de $169^{\circ} \mathrm{C}$ e $17 \%(\mathrm{~m} / \mathrm{m})$ de maltodextrina.

A polpa de graviola integral apresentou acidez em torno de $0,74 \%$ de ácido málico, pH por volta de 3,50, teor de sólidos solúveis ao redor de 9,6Brix e teor de vitamina
C de $37 \mathrm{mg} / 100 \mathrm{~g}$. Resultados semelhantes são relatados em outros trabalhos (LIMA et al., 2010; COSTA et al., 2014). Ainda com relação aos resultados encontrados, verificou-se que todos estavam de acordo com os teores preconizados pelos padrões de identidade e qualidade para este produto (BRASIL, 2000).

Observou-se também incremento nos valores de acidez e no teor de vitamina $C$ da polpa de graviola em pó, quando comparados com os valores da polpa integral, fato relacionado ao processo de secagem e, consequentemente, à concentração dos nutrientes no produto seco.

A polpa de graviola em pó apresentou coloração branca, com base nos baixos valores nos parâmetros $\mathrm{a}^{*}$, $\mathrm{b}^{\star}, \mathrm{C}^{*}$, e elevado valor no parâmetro $\mathrm{H}_{0}{ }^{*}$. A luminosidade da polpa de graviola integral e em pó foi de 67,22 e 67,06, respectivamente.

Produtos ricos em açúcares, como as polpas de frutas, podem sofrer escurecimento quando expostos a altas temperaturas durante os processos de secagem, resultando numa redução dos valores de luminosidade $\left(L^{*}\right)$. Esse comportamento foi relatado por Quek et al. (2007), em seu estudo com suco de melancia em pó, e por Rocha et al. (2014), em seu estudo com suco de caju em pó, ambos obtidos em spray dryer.

No presente trabalho, o fato de a luminosidade não ter sofrido alteração durante o processo pode ser visto como um aspecto positivo das condições de secagem utilizadas, indicando que não houve escurecimento do pó. Resultado semelhante foi relatado por Rocha et al. (2013), em estudo sobre a obtenção de polpa de manga em pó por spray dryer, e por Costa et al. (2014), estudando a influência da concentração de maltodextrina sobre as características físico-químicas da polpa de graviola em pó, obtida por secagem em spray dryer.

Tabela 3. Caracterização físico-química da polpa de graviola integral (base úmida) e em pó (base seca), obtida por secagem em spray dryer à temperatura de $169^{\circ} \mathrm{C}$ e $17 \%(\mathrm{~m} / \mathrm{m})$ de maltodextrina.

\begin{tabular}{lccc}
\multicolumn{1}{c}{ Parâmetro } & & Polpa integral (b.u.) & Polpa em pó (b.s.) \\
\hline Acidez titulável (\% m/m de ácido cítrico) & & $0,70 \pm 0,02$ & $2,53 \pm 0,15$ \\
(\% m/m de ácido málico) & & $0,74 \pm 0,03$ & $2,65 \pm 0,16$ \\
pH & & $3,50 \pm 0,03$ & $3,67 \pm 0,03$ \\
Sólidos solúveis totais ('Brix) & & $9,63 \pm 0,55$ & $*$ \\
Vitamina C (mg ácido ascórbico $100 \mathrm{~g}^{-1}$ ) & $\mathrm{L}^{*}$ & $37,79 \pm 0,09$ & $103,37 \pm 10,12$ \\
& $\mathrm{a}^{*}$ & $67,22 \pm 0,07$ & $-2,40 \pm 0,01$ \\
& $\mathrm{~b}^{*}$ & $-1,13 \pm 0,05$ & $6,11 \pm 0,01$ \\
Parâmetros de cor & $\mathrm{H}^{*}$ & $11,04 \pm 0,02$ & $111,40 \pm 0,08$ \\
& $\mathrm{C}^{*}$ & $95,55 \pm 0,02$ & $6,56 \pm 0,01$ \\
Umidade (\% m/m) & & $11,08 \pm 0,02$ & $1,33 \pm 0,20$ \\
Atividade de água & & $89,29 \pm 1,88$ & $0,15 \pm 0,01$ \\
Densidade aparente & & $0,98 \pm 0,01$ & $0,53 \pm 0,02$ \\
\hline
\end{tabular}

*Análise não aplicada ao material devido ao seu estado físico. 
A secagem à temperatura de $160^{\circ} \mathrm{C}$ e $17 \%(\mathrm{~m} / \mathrm{m})$ de maltodextrina como coadjuvante de secagem resultou em um pó com teor de umidade de 1,3\% e atividade de água de 0,1 . Isto significa que a polpa de graviola em pó pode ser considerada estável. No entanto, as condições de armazenamento irão desempenhar importante papel em sua estabilidade. As polpas de frutas em pó, devido ao elevado teor de açúcares, podem apresentar elevada higroscopicidade e devem ser armazenadas adequadamente em embalagens com barreira a troca de gases e umidade, bem como mantidas em ambiente fresco e seco.

A densidade aparente da polpa de graviola em pó foi de $0,53 \mathrm{~g} / \mathrm{mL}$. A baixa densidade está associada ao baixo teor de umidade do material e ao uso de maltodextrina. A adição de maltodextrina aumenta o teor de sólidos totais e reduz o teor de umidade do material a ser desidratado, facilitando a obtenção de pós com menor teor de umidade (QUEK et al., 2007). Pós com menor teor de umidade tendem a ser menos densos que pós com maior teor, uma vez que há menor presença de água em sua composição.

$\mathrm{Na}$ Tabela 4, encontram-se os resultados das análises higroscópicas no que concerne aos parâmetros de higroscopicidade, grau de caking, solubilidade e tempo de reidratação para a polpa em pó obtida a $169{ }^{\circ} \mathrm{C}$ com $17 \%(\mathrm{~m} / \mathrm{m})$ de maltodextrina.

A higroscopicidade da polpa de graviola em pó foi de 10,41\%. De acordo com a classificação proposta por GEA Niro Research Laboratory (2003), a polpa de graviola em pó obtida nas condições otimizadas de secagem é ligeiramente higroscópica. A ligeira higroscopicidade da polpa de graviola em pó sugere a necessidade de uma investigação mais profunda sobre seu comportamento higroscópico durante seu armazenamento.

A polpa de graviola em pó apresentou o valor de $51,88 \%$ para o parâmetro de grau de caking, podendo ser classificado como pó com muita formação de caking, de acordo com a classificação proposta por GEA Niro Research Laboratory (2003). Segundo Goula e Adamopoulos (2010), o caking de um pó alimentício depende de sua higroscopicidade, uma vez que sua formação em pós alimentícios ricos em açúcares pode ser atribuída à absorção de umidade. A água é adsorvida na superfície das partículas, formando uma solução saturada e tornando as partículas pegajosas.

A solubilidade da polpa de graviola em pó (55,74\%) revela seu potencial como ingrediente em produtos

Tabela 4. Parâmetros higroscópicos da polpa de graviola em pó

\begin{tabular}{ll}
\multicolumn{1}{c}{ Parâmetro } & Polpa em pó \\
Higroscopicidade (\%) & $10,41 \pm 1,30$ \\
Grau de caking (\%) & $51,85 \pm 1,47$ \\
Solubilidade (\%) & $55,74 \pm 3,24$ \\
Tempo de reidratação (s) & $10,33 \pm 0,58$ \\
\hline
\end{tabular}

alimentícios, como bebidas e preparados de sobremesas instantâneos.

O tempo de reidratação médio para a polpa de graviola em pó foi de 10,3 segundos, o qual é considerado relativamente rápido (GOULA; ADAMOPOULOS, 2010). A rápida reidratação é possivelmente explicada pela alta concentração de maltodextrina, uma vez que a mesma apresenta elevada solubilidade (CANO-CHAUCA et al., 2005). A utilização de maltodextrina resulta ainda em pós com baixo teor de umidade, contribuindo para uma rápida reidratação, pois, quanto menor o teor de umidade, menos pegajoso é o pó e, portanto, maior será a superfície da área em contato com a água de hidratação (GOULA; ADAMOPOULOS, 2008).

As partículas da polpa de graviola em pó obtida no estudo pelo processo de secagem em spray dryer a $169{ }^{\circ} \mathrm{C}$ com $17 \%(\mathrm{~m} / \mathrm{m})$ de maltodextrina apresentaram formato esférico, de diversos tamanhos, superfície lisa e uniforme (Figura 2), características desejáveis para a estabilidade do material (OSORIO et al., 2010) e atribuídas ao uso de maltodextrina e à temperatura do ar de entrada utilizada no processo de secagem.

Loksuwan (2007) relata que a maltodextrina com 20DE contém uma grande quantidade de açúcares de baixo peso molecular, que podem atuar como plastificantes, evitando o encolhimento da superfície durante a secagem em spray dryer e conduzindo à formação de partículas mais lisas. Caparino et al. (2012) observaram estruturas semelhantes, de superfície fina e lisa, para a polpa de manga em pó contendo $25 \%$ de maltodextrina e obtida por secagem em spray dryer.

Nijdam e Langrish (2006) relataram que o uso de temperaturas mais altas no processo de secagem em spray dryer promove rápida evaporação da água e a película do material desidratado torna-se seca e rígida, impedindo que as partículas encolham; assim, como resultado, temos uma estrutura mais uniforme. Tais conclusões foram obtidas ao observarem a formação de esferas mais lisas e rígidas em leite em pó obtido por secagem em spray dryer, à temperatura de $200^{\circ} \mathrm{C}$, enquanto que o leite produzido a $120^{\circ} \mathrm{C}$ apresentou partículas menores e com aparência mais rugosa.

Em alguns pontos da imagem, é possível observar a adesão de partículas menores na superfície de partículas maiores, formando aglomerados (Figura 2B). De acordo com Cano-Chauca et al. (2005), a forte aderência das partículas menores em torno das maiores demonstra a ausência de superfícies cristalinas e é característica de produtos amorfos. A formação de aglomerados contribui para os baixos valores de densidade aparente e para a classificação do pó como ligeiramente higroscópico e de muita formação de caking. 
A.

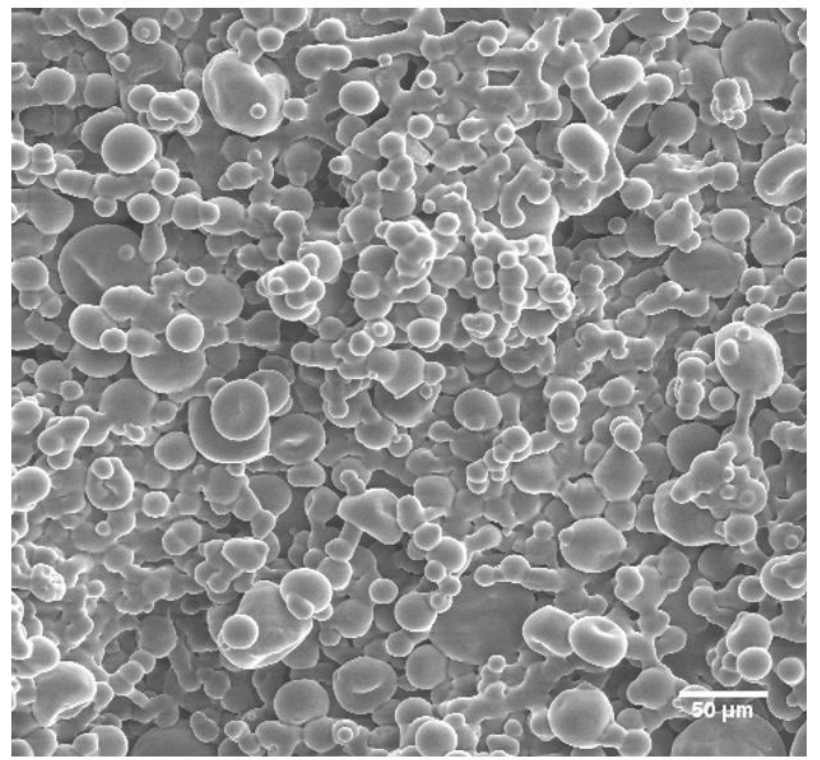

B.

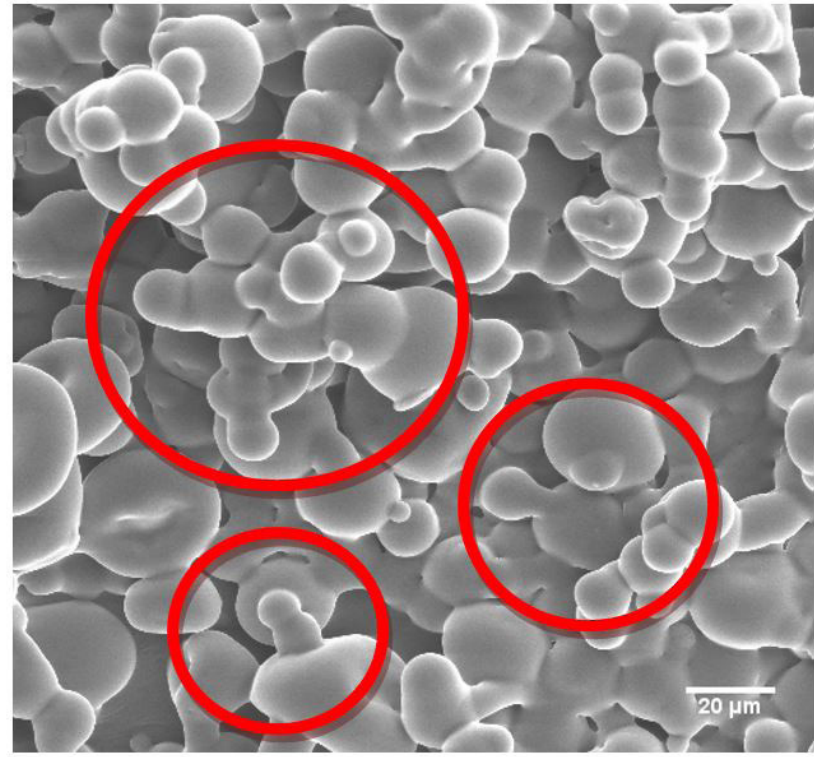

Figura 2. Imagens microscópicas obtidas por microscopia eletrônica de varredura (MEV), com aumento de 200 vezes - Barra = 50 um (A) e aumento de 500 vezes - Barra $=20 \mu \mathrm{m}(\mathrm{B})$, da polpa de graviola em pó obtida por secagem em spray dryer, à temperatura de $169{ }^{\circ} \mathrm{C}$ e $17 \%(\mathrm{~m} / \mathrm{m})$ de maltodextrina.

A polpa de graviola em pó obtida por secagem em spray dryer pode ser considerada um pó de qualidade, pois apresentou baixo teor de umidade, atividade de água e higroscopicidade, além de considerada solubilidade e rápido tempo de reidratação. Tais características são comumente descritas como importantes para alimentos em pós (BAKAR et al., 2013).

\section{Conclusão}

A secagem da polpa de graviola em spray dryer a $169{ }^{\circ} \mathrm{C}$ e com adição de $17 \%(\mathrm{~m} / \mathrm{m})$ de maltodextrina, como agente carreador, propiciou maior rendimento e pós com características físico-químicas e higroscópicas consideradas boas para pós alimentícios.

\section{Referências}

ABADIO, F. D. B.; DOMINGUES, A. M.; BORGES, S. V.; OLIVEIRA, V. M. Physical properties of powdered pineapple (Ananás comosus) juice-effect of maltodextrin concentration and atomization speed. Journal of Food Engineering, v. 64, n. 3, p. 285-287, 2004. http://dx.doi.org/10.1016/j.jfoodeng.2003.10.010.

BAKAR, J.; EE, S. C.; MUHAMMAD, K.; HASHIM, D. M.; ADZAHAN, N. Spray-drying optimization for red pitaya peel (Hylocereus polyrhizus). Food and Bioprocess Technology, v. 6, n. 5, p. 1332-1342, 2013. http://dx.doi.org/10.1007/s11947-012-0842-5.

BRASIL. Ministério da Agricultura, Pecuária e Abastecimento. Instrução normativa $n^{\circ} 1$, de 7 de janeiro de 2000. Dispõe sobre o "Regulamento Técnico geral para fixação dos padrões de identidade e qualidade para polpa de fruta". Diário Oficial da União, Brasília, DF, 7 jan. 2000.
CANO-CHAUCA, M.; STRINGHETA, P. C.; RAMOS, A. M.; CAL-VIDAL, $\mathrm{J}$. Effect of the carriers on the microstructure of mango powder obtained by spray drying and its functional characterization. Innovative Food Science \& Emerging Technologies, v. 6, n. 4, p. 420-428, 2005. http://dx.doi.org/10.1016/j.ifset.2005.05.003.

CAPARINO, O. A.; TANG, J.; NINDO, C. I.; SABLANI, S. S.; POWERS, J. R.; FELLMAN, J. K. Effect of drying methods on the physical properties and microstructures of mango (Philippine 'Carabao' var.) powder. Journal of Food Engineering, v. 111, n. 1, p. 135-148, 2012. http://dx.doi.org/10.1016/j.jfoodeng.2012.01.010.

COSTA, J. P.; ROCHA, É. M. F. F.; COSTA, J. M. C. Study of the physicochemical characteristics of soursop powder obtained by spray-drying. Food Science and Technology, v. 34, n. 4, p. 663-666, 2014. http://dx.doi.org/10.1590/1678-457X.6380.

CUNNIFF, P. (Ed.). Official methods of analysis of the Association of Official Analytical Chemists. Washington: AOAC, 1997. v. 2.

FERRARI, C. C.; RIBEIRO, C. P.; AGUIRRE, J. M. Secagem por atomização de polpa de amora-preta usando maltodextrina como agente carreador. Brazilian Journal of Food Technology, v. 15, n. 2, p. 157-165, 2012. http://dx.doi.org/10.1590/S198167232012005000009 .

GEA NIRO RESEARCH LABORATORY. Analytical methods dry milk products. Soeborg: GEA Niro, 2003. 103 p. (GEA Niro Analytical Methods 14a-15a).

GOULA, A. M.; ADAMOPOULOS, K. G. A new technique for spray drying orange juice concentrate. Innovative Food Science \& Emerging Technologies, v. 11, n. 2, p. 342-351, 2010. http:// dx.doi.org/10.1016/j.ifset.2009.12.001. 
Avaliação dos parâmetros de secagem da polpa de graviola em pó obtida por secagem em spray dryer

Cavalcante, C. E. B. et al.

GOULA, A. M.; ADAMOPOULOS, K. G. Effect of maltodextrin addition during spray drying of tomato pulp in dehumidified air: I. powder properties. Drying Technology, v. 26, n. 6, p. 726-737, 2008. http://dx.doi.org/10.1080/07373930802046377

INSTITUTO ADOLFO LUTZ - IAL. Normas analíticas do Instituto Adolfo Lutz: métodos químicos e físicos para análise de alimentos. 4. ed. São Paulo: Instituto Adolfo Lutz, 2005. 1020 p.

JAYA, S.; DAS, H. Effect of maltodextrin, glycerol monostearate and tricalcium phosphate on vaccum dried mango powders properties. Journal of Food Engineering, v. 63, n. 2, p. 125-134, 2004. http://dx.doi.org/10.1016/S0260-8774(03)00135-3.

KHA, T. C.; NGUYEN, M. H.; ROACH, P. D. Effects of spray drying conditions on the physicochemical and antioxidant properties of the gac (Mormodica cochinchinesis) fruit aril powder. Journal of Food Engineering, v. 98, n. 3, p. 385-392, 2010. http://dx. doi. org/10.1016/j.jfoodeng.2010.01.016.

LEMOS, E. E. P. A produção de anonáceas no Brasil. Revista Brasileira de Fruticultura, v. 36, p. 78-85, 2014. Edição especial.

LIMA, M. A. C.; ALVES, R. E.; FILGUEIRAS, H. A. C. Comportamento respiratório e amaciamento da graviola (Annona muricata L.) após tratamentos pós-colheita com cera e 1-metilciclopropeno. Ciência e Agrotecnologia, v. 34, n. 1, p. 155-162, 2010. http:// dx.doi.org/10.1590/S1413-70542010000100020.

LOKSUWAN, J. Characteristics of microencapsulated $\beta$-carotene formed by spray drying with modified tapioca starch, native tapioca starch and maltodextrin. Food Hydrocolloids, v. 21, n. 5-6, p. 928-935, 2007. http://dx.doi.org/10.1016/j.foodhyd.2006.10.011.

MOREIRA, G. E. G.; COSTA, M. G. M.; SOUZA, A. C. R.; BRITO, E. S.; MEDEIROS, M. Physical properties of spray dried acerola pomace extract as affected by temperature and drying aids. Lebensmittel-Wissenschaft + Technologie, v. 42, n. 2, p. 641-645, 2009. http://dx.doi.org/10.1016/j.Iwt.2008.07.008.

NIJDAM, J. J.; LANGRISH, T. A. J. The effect of surface composition on the functional properties of milk powders. Journal of Food Engineering, v. 77, n. 4, p. 919-925, 2006. http://dx. doi. org/10.1016/j.jfoodeng.2005.08.020.

OLIVEIRA, A. R. G.; BORGES, S. V.; FARIA, R. K.; ENDO, E.; GREGÓRIO, S. R. Influência das condições de secagem por atomização sobre as características sensoriais de sucos maracujá (Passiflora edulis) e abacaxi (Ananas comosus) desidratados. Revista Ciência Agronômica, v. 38, n. 3, p. 251-256, 2007.
OSORIO, C.; ACEVEDO, B.; HILLEBRAND, S.; CARRIAZO, J.; WINTERHALTER, P.; MORALES, A. L. Microencapsulation by spray drying of anthocyanin pigments from corozo (Bactris guineensis) fruit. Journal of Agricultural and Food Chemistry, v. 58, n. 11, p. 6977-6985, 2010. PMid:20459133. http://dx.doi. org/10.1021/jf100536g.

QUEK, S. Y.; CHOK, N. K.; SWEDLUND, P. The physicochemical properties of spray-dried watermelon powder. Chemical Engineering and Processing, v. 46, n. 5, p. 386-392, 2007. http://dx.doi.org/10.1016/j.cep.2006.06.020.

ROCHA, É. M. F. F.; MOURA NETO, L. G.; RODRIGUES, S.; AFONSO, M. R. A.; COSTA, J. M. C. Influence of atomization parameters in the obtention of mango pulp powder. Journal of Food Science and Engineering, n. 3, p. 357-362, 2013.

ROCHA, É. M. F. F.; SOUSA, S. L.; COSTA, J.; RODRIGUES, S.; AFONSO, M. R. A.; COSTA, J. M. C. Obtenção de suco de caju atomizado através do controle das condições de secagem. Revista Brasileira de Engenharia Agrícola e Ambiental, v. 18, n. 6, p. 646-651, 2014. http://dx.doi.org/10.1590/S141543662014000600012.

SÃO JOSÉ, A. R.; PIRES, M.M.; FREITAS, A. L. G. E.; RIBEIRO, D. P.; PEREZ, L. A. A. Atualidades e perspectivas das anonáceas no mundo. Revista Brasileira de Fruticultura, v. 36, p. 86-93, 2014. Edição especial.

STATSOFT. Statistic for Windows. versão 7.0. Tulsa: StatSoft Inc., 2007.

TONON, R. V.; BARONI, A. F.; BRABET, C.; GIBERT, O.; PALLET, D.; HUBINGER, M. D. Water sorption and glass transition temperature of spray-dried açai (Euterpe oleracea Mart.) juice. Journal of Food Engineering, v. 94, n. 3-4, p. 215-221, 2009. http://dx.doi.org/10.1016/j.jfoodeng.2009.03.009.

TONON, R. V.; BRABET, C.; HUBINGER, M. D. Influence of process conditions on the physicochemical properties of açai (Euterpe oleraceae Mart.) powder produced by spray drying. Journal of Food Engineering, v. 88, n. 3, p. 411-418, 2008. http://dx.doi.org/10.1016/j.jfoodeng.2008.02.029.

WATANABE, H. S.; OLIVEIRA, S. L.; CAMARA, F. M.; ALMEIDA, G. V. B.; ALVES, A. A. Perfil de comercialização das anonáceas nas Ceasas brasileiras. Revista Brasileira de Fruticultura, v. 36, p. 65-70, 2014. Edição especial. 